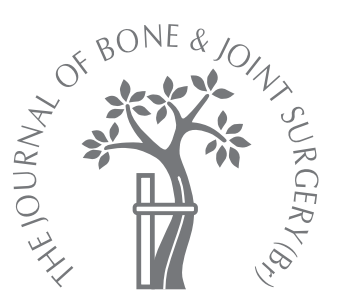

G. S. Biring, A. Hashemi-Nejad, A. Catterall

From the Royal National Orthopaedic Hospital, Stanmore, England

\title{
Outcomes of subcapital cuneiform osteotomy for the treatment of severe slipped capital femoral epiphysis after skeletal maturity
}

\begin{abstract}
We reviewed prospectively, after skeletal maturity, a series of 24 patients (25 hips) with severe acute-on-chronic slipped capital femoral epiphysis which had been treated by subcapital cuneiform osteotomy. Patients were followed up for a mean of 8 years, 3 months ( 2 years, 5 months to 16 years, 4 months). Bedrest with 'slings and springs' had been used for a mean of 22 days (19 to 35 ) in 22 patients, and bedrest alone in two, before definitive surgery. The lowa hip score, the Harris hip score and Boyer's radiological classification for degenerative disease were used.

The mean lowa hip score at follow-up was 93.7 (69 to 100) and the mean Harris hip score 95.6 (78 to 100). Degenerative joint changes were graded as 0 in 19 hips, grade 1 in four and grade 2 in two. The rate of avascular necrosis was $12 \%$ (3 of 25) and the rate of chondrolysis was $16 \%$ (4 of 25). We conclude that after a period of bed rest with slings and springs for three weeks to gain stability, subcapital cuneiform osteotomy for severe acute-on-chronic slipped capital femoral epiphysis is a satisfactory method of treatment with an acceptable rate of complication.
\end{abstract}

The treatment of severe slipped capital femoral epiphysis (SCFE) is controversial and often difficult. ${ }^{1}$ Severe cases comprise approximately $5 \%$ to $10 \%$ of all cases of SCFE, ${ }^{2-4}$ and in general the long-term results of these slips treated by any method are poor. ${ }^{2,5-8}$

The aims of treatment are to prevent further epiphyseal displacement, to avoid complications such as avascular necrosis and chondrolysis, to correct the deformity and to maintain hip function. The importance of SCFE in the aetiology of osteoarthritis of the hip in the adult has been recognised. ${ }^{9,10}$ This raises the question as to whether reduction of the deformity can reduce the severity, and delay the onset, of symptoms in adults.

Possible treatments available for severe SCFE are pinning in situ, epiphysiodesis or proximal femoral osteotomy. The last can be performed at the physis, ${ }^{11,12}$ at the base of the femoral neck ${ }^{13,14}$ or at the intertrochanteric/ subtrochanteric level. ${ }^{15,16}$ Acetabular development is almost complete at presentation in this group of patients and therefore the potential for acetabular remodelling is limited. The metaphysis may impinge on the lateral rim of the acetabulum and lead to accelerated degeneration. The purpose of a subcapital cuneiform osteotomy as described by Fish ${ }^{12}$ is to correct this severe deformity at the time of initial treat- ment since it is unlikely to remodel. The osteotomy restores the normal relationship of the head, neck and shaft, thereby potentially improving kinematics and preventing or delaying the onset of osteoarthritis. Such a joint would be expected to function normally in the short and long term.

Our aim was to review the clinical and radiological results of subcapital cuneiform osteotomy at skeletal maturity.

\section{Patients and Methods}

Between 1989 and 2003, 26 patients underwent subcapital cuneiform osteotomy at our institution. Two were lost to follow-up. A series of 25 hips in 24 patients was therefore available for prospective clinical and radiological review at a mean follow-up of eight years three months $(2$ years 5 months to 16 years 4 months).

There were 15 boys and nine girls with a mean age at presentation of 13.8 years ( 11.3 to 15.7). Girls presented earlier, at a mean of 12.1 years (11.3 to 13.6$)$, than boys at 14.8 years (13.3 to 15.7$)$. The mean age at follow-up was 21.5 years (14 to 34$)$. There were 13 left hips and 12 right. One patient had hypothyroidism.

The slip was classified into categories based on duration of symptoms (acute, acute-onchronic or chronic), the angle of slip (mild, 


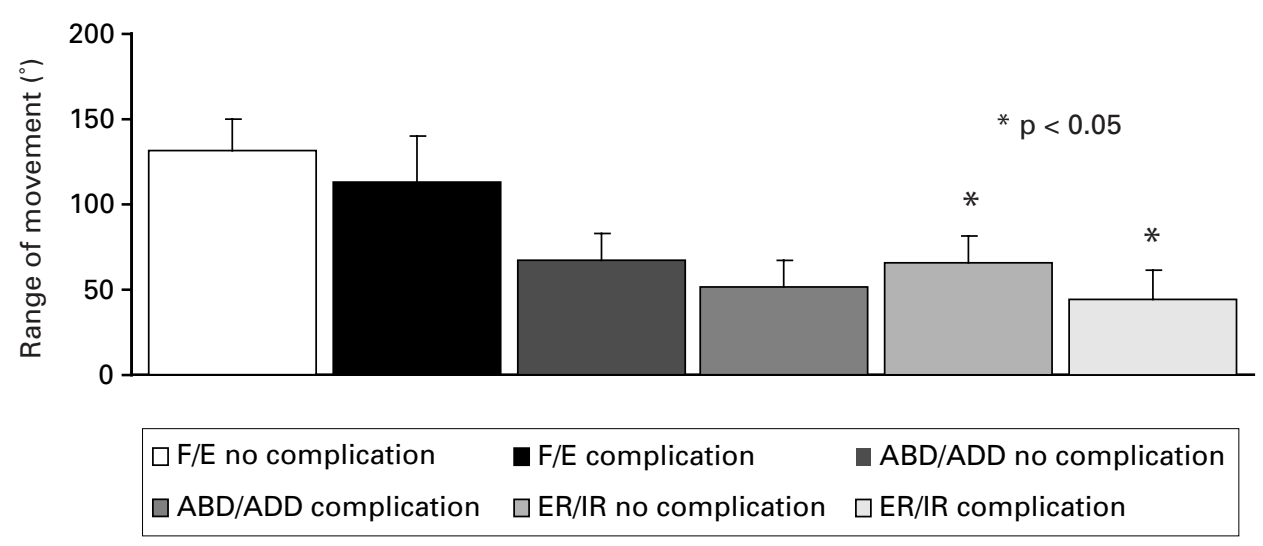

Fig. 1

The mean range of arc of movement $( \pm S D)$ for those with and without complications (avascular necrosis and chondrolysis). F/E, flexion/extension; ABD/ADD, abduction/adduction; ER/IR, external rotation/internal rotation.

moderate or severe), and the degree of stability (stable or unstable) based on the classification of Loder et al. ${ }^{17}$

Standard anteroposterior and lateral radiographs of both proximal femora were obtained before and after surgery and the angle of the slip was measured using the technique of Southwick. ${ }^{15}$ A severe slip was that greater than $60^{\circ}$. Functional outcome was assessed using the Iowa hip score ${ }^{18}$ and the Harris hip score (HHS).${ }^{19}$ Radiological classification of degenerative joint disease according to Boyer et $\mathrm{al}^{3}$ was measured at follow-up.

Patients with an Iowa hip score of between 90 and 100 points were rated as excellent, good with 80 to 89 points, fair with 70 to 79 points and poor with less than 70 points. ${ }^{18}$ Using the HHS, a score of $\geq 90$ points was classified as excellent and $<90$ as fair or poor. ${ }^{20}$

Pre-operative regime. Patients undergoing subcapital cuneiform osteotomy were treated by bed rest on slings and springs for a mean period of 22 days (19 to 35) before operation. This is a method of resting the lower limb in a sling suspended by a spring attached above the bed to support the weight of the limb in the position of comfort. The rationale for this treatment was to allow the joint effusion to settle and the blood supply to the epiphysis to recover. After resting the limb for three weeks in this way the patient was able to move the hip and the limb actively against the resistance of the spring indicating that the acute severe unstable slip had become stable and free from pain.

Operative technique. The subcapital cuneiform osteotomy described by Fish ${ }^{12}$ was performed through a Watson-Jones approach, ${ }^{21}$ whereas we preferred to use an anterior approach to the hip. The osteotomy was performed by removing a wedge of bone from the femoral neck at the level of the physis with gentle release of the tight inferomedial structures. The osteotomy shortened the femoral neck, allowing reduction of the epiphysis onto the metaphysis without tension using a Griffith-type manoeuvre. ${ }^{22}$ Under radiological control two percutaneous cannulated screws were inserted from a lateral approach to maintain reduction. The wounds were then closed. Post-operatively, patients were again treated by slings and springs until they had achieved satisfactory hip control with active flexion and abduction of the hip. They were then mobilised, 'toetouch' weight-bearing for eight weeks until the osteotomy had healed. Hydrotherapy was used as part of the rehabilitation regime.

Statistical analysis. The Mann-Whitney U test (two-tailed test) was used to compare mean values. A p-value $<0.05$ was considered to be significant.

\section{Results}

All hips were classified as acute-on-chronic, and all as severe with a mean slip angle of $74^{\circ}\left(60^{\circ}\right.$ to $\left.90^{\circ}\right)$. All but two were categorised as unstable. These two patients were not treated in slings and springs, but had been on bedrest in the referring hospital for three weeks before transfer and were therefore considered to be stable before surgery. The mean time from the onset of symptoms to acute presentation was 5.7 months (1 to 18 ).

The mean Iowa hip score was 93.7 (69 to 100 ). There were 22 hips rated as excellent, one good, one fair and one as poor. The mean HHS was 95.6 (78 to 100) and 22 hips were rated as excellent and three as fair/poor. The mean flexion-extension arc was $126^{\circ}\left(75^{\circ}\right.$ to $\left.140^{\circ}\right)$, the mean abduction-adduction arc $62^{\circ}\left(40^{\circ}\right.$ to $\left.80^{\circ}\right)$ and the mean external rotation-internal rotation arc $60^{\circ}\left(20^{\circ}\right.$ to $\left.80^{\circ}\right)$. When hips with or without complications were compared, only the mean external rotation-internal rotation arc was significantly higher in those without complications $(\mathrm{p}<$ 0.05) (Fig. 1).

Shortening of a mean of $1.2 \mathrm{~cm} \mathrm{(0.5} \mathrm{to} \mathrm{2.5)} \mathrm{was} \mathrm{present}$ in 20 patients. Only one patient required a shoe-raise. No patient had a Trendelenburg gait. ${ }^{23}$

According to the classification of Boyer et $\mathrm{al}^{3}$ the mean radiological degenerative grade was 0.3 . Grade 0 degener- 


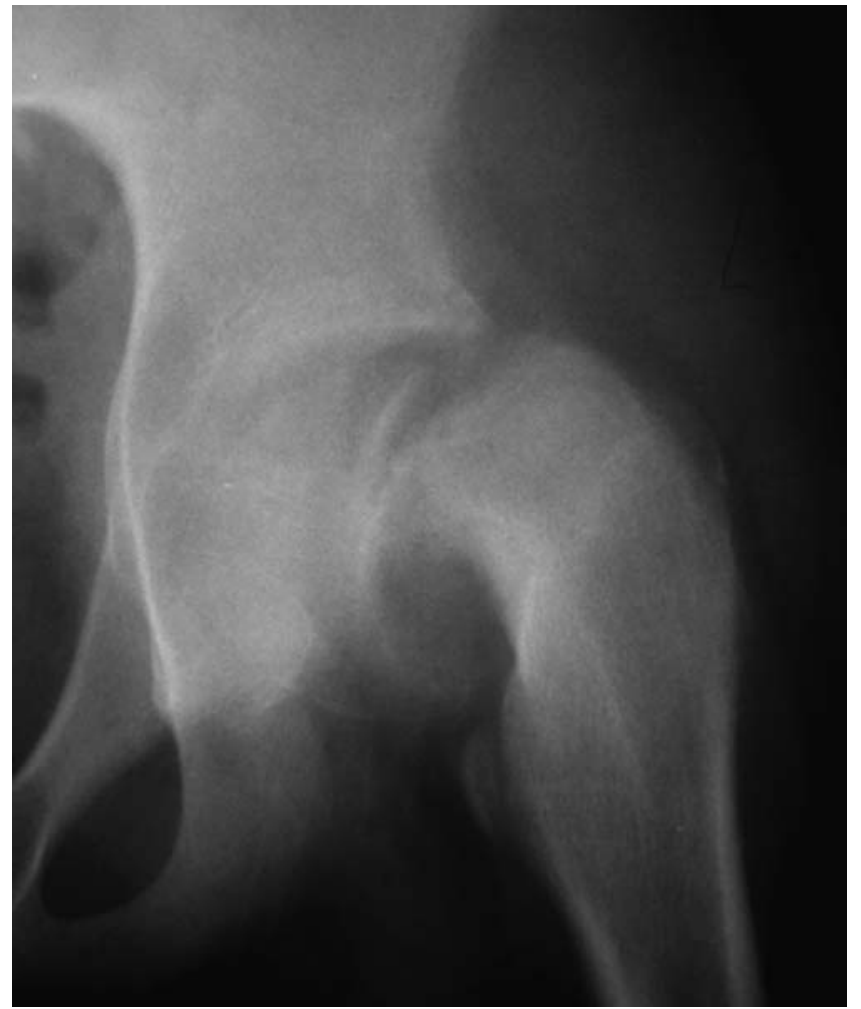

Fig. 2a

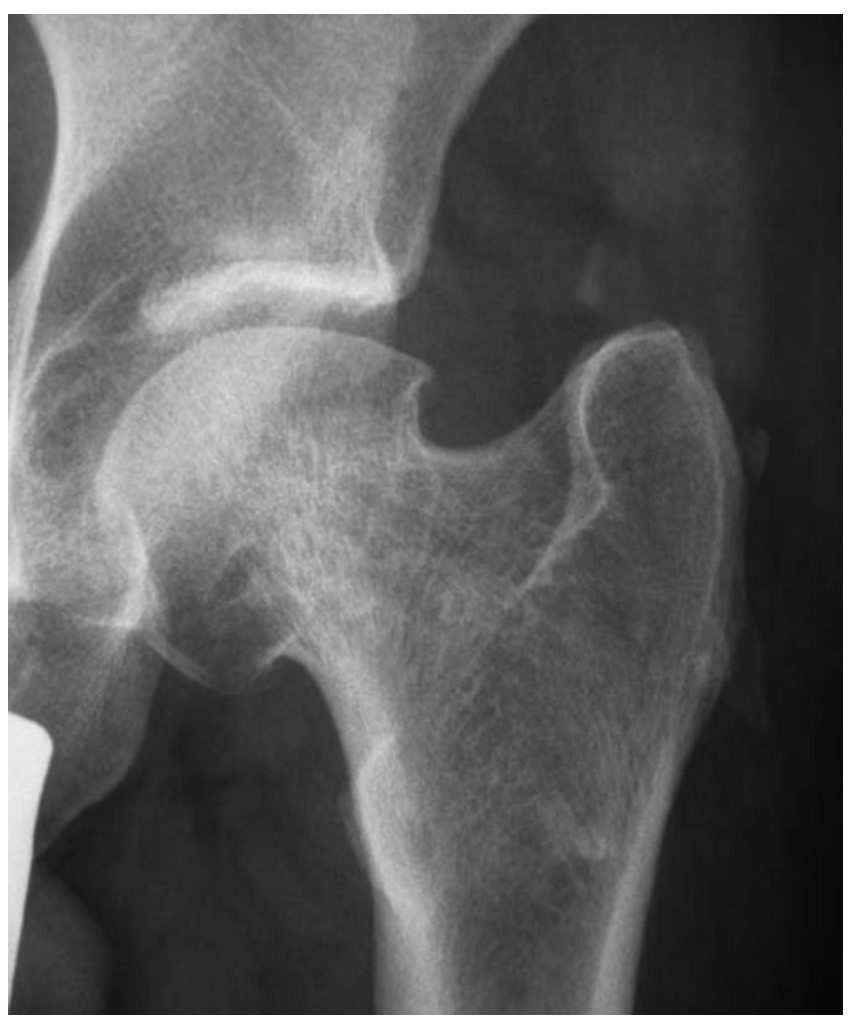

Fig. 2c

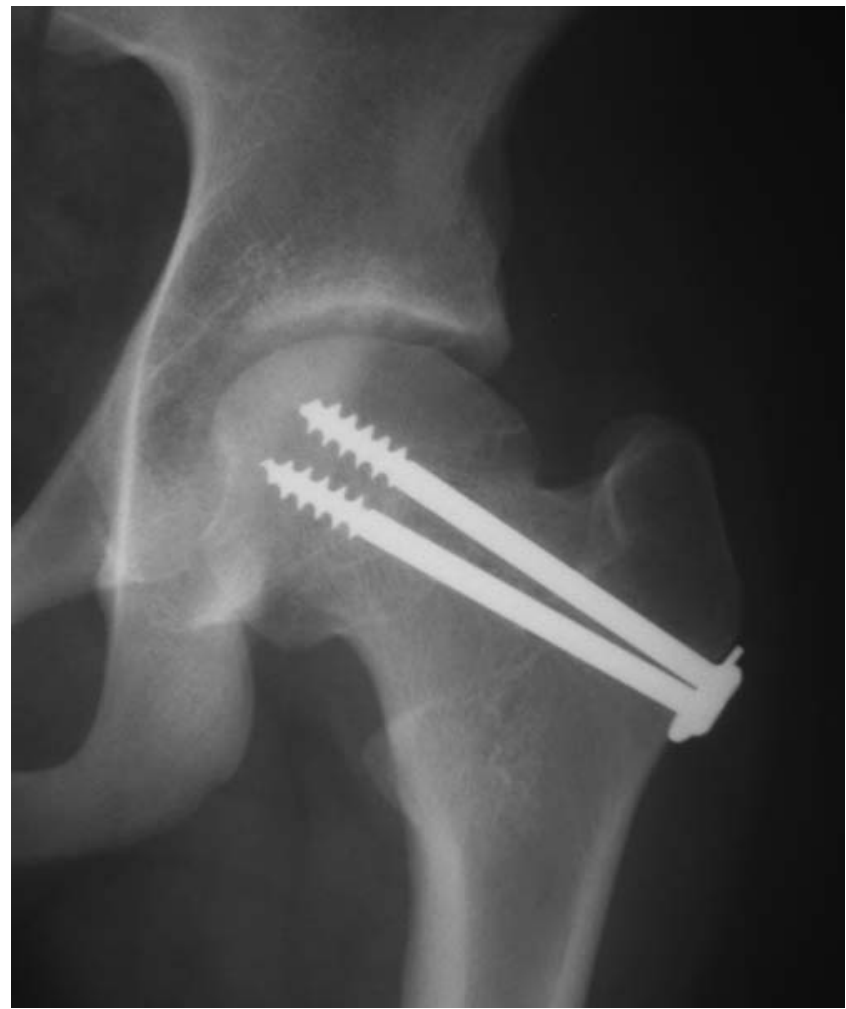

Fig. $2 b$

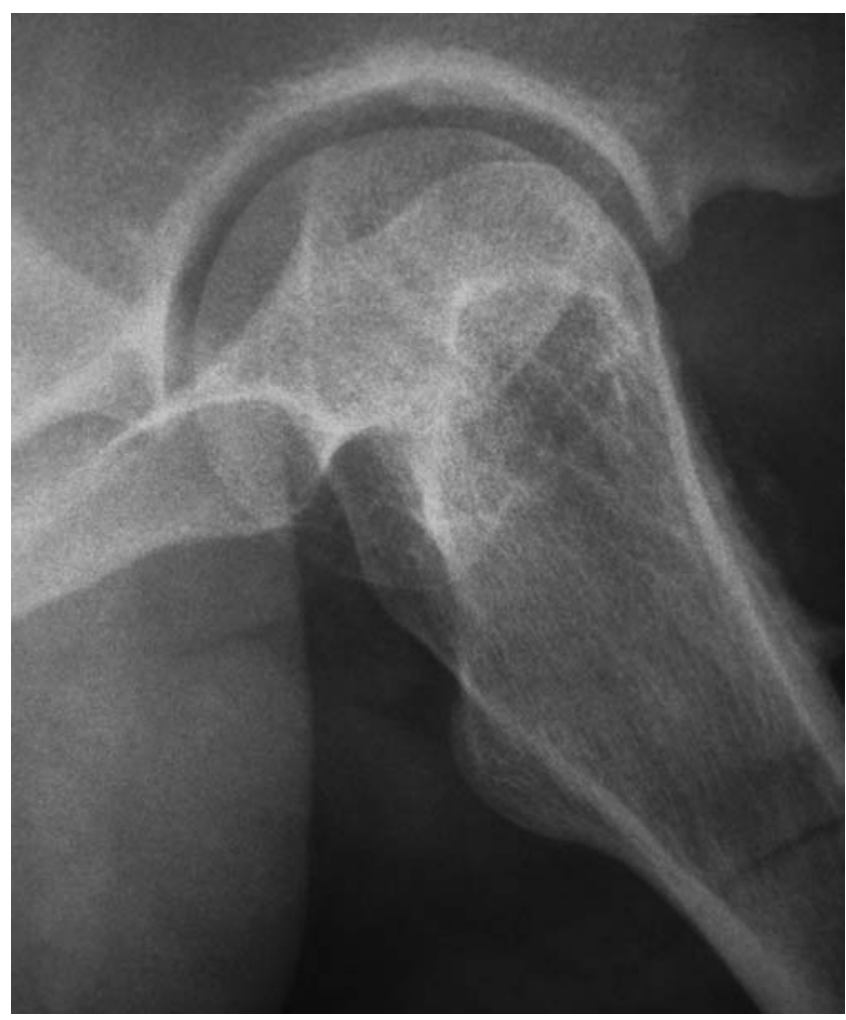

Fig. $2 d$

Radiographs of a patient with a severe, unstable, acute-on-chronic slipped capital femoral epiphysis, showing a) pre-operative anteroposterior view, b) immediate post-operative anteroposterior view, and c,d) anteroposterior and lateral radiographs after removal of the metalwork 15 years later. 
ative changes were present in 19 hips, grade 1 in four and grade 2 in two. Of these, 23 hips $(92 \%)$ had a normal joint space and a mean Iowa hip score of 95.0 (78 to 100). Two $(8 \%)$ with an abnormal joint space (Boyer grade 2 ) had a mean Iowa hip score of 79.0 (75 to 83). The mean HHS for hips with a normal joint space was 96.0 (78 to 100) and for those with Boyer grade- 2 changes 93.0 (90 to 96). Both patients with grade 2 changes had complications. One had avascular necrosis (AVN) and the other chondrolysis. The radiographs of a patient with an excellent score and grade 0 changes at 15 years are shown in Figure 2.

There were no cases of wound infection, neurovascular injury or deep-vein thrombosis.

Four hips $(16 \%)$ developed chondrolysis. All were diagnosed within the first six months. Two cases were transient and had complete recovery within 18 months with excellent functional scores (both had an Iowa hip score of 96.5 and the mean HHS was 97 (94 to 100)). The remaining two patients had a mean Iowa hip score of 71.9 (69 to 75) and a mean HHS of 84.5 (78 to 90) at follow-up. One had grade 1 and one grade 2 degenerative changes as seen on radiographs. The Iowa hip score and HHS for the four patients with chondrolysis did not differ significantly from those without this complication $(\mathrm{p}=0.06)$.

Three cases of AVN (12\%) were diagnosed within one year of operation. Two patients had partial segmental avascular necrosis and the third had complete involvement of the femoral head. The mean Iowa hip score and HHS were 88.0 (82 to 92 ) and 92.6 (84 to 98), respectively. The numbers were small, but there was a trend for those with AVN to have lower scores. The patient with complete involvement of the femoral head had removal of their screws at one year and then underwent a lateral shelf acetabuloplasty 18 months after the first operation, followed by a femoral osteotomy 4.5 years after the initial treatment. This patient had grade 2 degenerative changes.

When comparing the mean Iowa hip score and HHS in patients with and without complications, significantly higher scores were found in those without complications (chondrolysis $\mathrm{p}=0.01 ; \mathrm{AVN} \mathrm{p}=0.05$ ).

No immediate additional operations were needed, but six patients required removal of screws at a mean of 24 months (6 to 34). One of these required a contralateral distal femoral epiphysiodesis at the same time. The patient with the longest follow-up and a slip angle of $70^{\circ}$ had been treated initially at a local hospital by two attempts at pinning in situ which had been unsuccessful. He now enjoys excellent functional scores with no evidence of degenerative changes (grade 0 ). No patient had ipsilateral knee, back or contralateral hip pain.

\section{Discussion}

For subcapital cuneiform osteotomy to be satisfactory it must be effective in terms of the clinical result with minimal complications. In our study 22 of the 24 patients (92\%) had an excellent result. There were three cases of AVN
$(12 \%)$ and four of chondrolysis (16\%), of which two were transient. The prognosis for a child with a stable SCFE is good with a low incidence of AVN. ${ }^{17}$ That for a child with an unstable SCFE is guarded because of the increased risk of AVN. The reported incidence ranges from $3 \%$ to $47 \%{ }^{8,17,24,25}$ Several authors have stated that the high complication rates in the unstable slip are most likely to be secondary to vascular injury caused at the time of initial displacement. ${ }^{17,26}$ Rattey et $\mathrm{al}^{8}$ showed that the Iowa hip score and the severity of the degenerative changes were related to the severity of the slip. Their findings suggested that the severity of the slip, independent of the presence of AVN, may affect the outcome and that malunion of a SCFE may have long-term consequences. This highlights the difficulty in determining whether the severity of the slip or the method of treatment is the main factor in determining outcome.

Without treatment the long-term prognosis for severe slips is poor, with degenerative changes occurring in most cases. ${ }^{2,3}$ Surgical intervention has been advocated by many, but no single technique has gained universal acceptance. Several authors have obtained acceptable results by treating severe slips with screw fixation in situ. ${ }^{27-29}$ However, others have stated that even with improved methods of imaging and fixation, the pinning of severe slips is extremely demanding and technical errors have been associated with chondrolysis, AVN and degenerative arthritis. ${ }^{30,31}$ Those authors who have advocated pinning in situ state that the hip has a capacity to remodel, ${ }^{32,33}$ but reports have been variable. Siegel et $\mathrm{al}^{34}$ used CT to show that despite remodelling of the metaphysis, there was little alteration of the relationship between the neck and femoral head.

It has been suggested that femoral metaphyseal impingement on the acetabulum accelerates the degenerative process. Corrective osteotomies aim to resolve this and can be performed at the site of the deformity at the level of the physis, at the base of the femoral neck or at the trochanteric level. The risk of complications is less at the base of the neck and with the use of a trochanteric osteotomy, but correction of the deformity is also less.

In the past, subcapital cuneiform osteotomy has been associated with a high rate of complications..$^{27,35}$ However, there have been several reports with a good outcome. ${ }^{11,12,36,37}$ These stressed the importance of meticulous technique while performing the osteotomy and the avoidance of forceful manipulation. Fish ${ }^{12}$ reported that $92 \%$ of his patients had good results with a rate of AVN of $4.5 \%$ and of chondrolysis of $3 \%$. This series included mild and moderate slips. DeRosa et $\mathrm{al}^{37}$ reported good results in $95 \%$ of patients with a rate of AVN of $15 \%$. Dunn and Angel ${ }^{11}$ reported excellent results in patients with severe chronic SCFE treated by subcapital cuneiform osteotomy. In their study, patients with acute-on-chronic slips had more complications with a rate of AVN of $39 \%$ and of chondrolysis of $17 \%$. They provided good evidence that femoral neck osteotomies should only be done for chronic 
and not acute slips because of the high risk of complications. The patients in our study were rested for three weeks in slings and springs to allow the circulation to the epiphysis to stabilise. This may explain the lower incidence of AVN and chondrolysis.

The literature with regard to the timing of the operation is controversial. Loder et $\mathrm{al}^{17}$ showed a rate of AVN of $88 \%$ in unstable hips treated within 24 hours and of $32 \%$ in those treated more than 48 hours after the onset of symptoms. Other studies have shown lower rates of AVN for patients with unstable hips which were reduced and stabilised within 24 hours compared with those whose intervention was delayed. ${ }^{25,38}$ Phillips et a ${ }^{38}$ reported that all 14 of their unstable slips treated within 24 hours had no complications. None of the unstable hips in their study had evidence of chronicity. If patients present with an acute severe unstable slip within 24 hours without evidence of chronicity we would advocate an attempt at gentle reduction and screw fixation. However, in our study, no patient presented within the first 24 hours from the onset of symptoms to our hospital, which is a tertiary referral centre, and all had acute-on-chronic slips. In the presence of the adaptive softtissue changes which take place in the capsule in chronic slips, we consider that it would be unwise to attempt early reduction and stabilisation to correct deformity because of the risk of complications by stretching the already contracted soft tissues and blood vessels. As a result, our unit has a policy of a three-week wait before embarking on surgical intervention if there is evidence of chronicity. The patients are rested on slings and springs without any traction, to allow the effusion to subside and the pain to settle. After three weeks, when the patient has satisfactory painfree control of the hip, we consider the circulation to the epiphysis to have stabilised and regard the slip as stable.

It is only in a severe chronic stable slip that we advocate subcapital cuneiform osteotomy. The mean delay in diagnosis of 5.7 months, which has been highlighted in the literature $\mathrm{e}^{39,40}$ is a cause for concern. If recognised and treated earlier, progression to a severe unstable slip with its associated morbidity may be prevented.

Controversy in the treatment of SCFE is now focused on the most risk-prone hips, in particular those which are unstable and severe. Our study describes our experience with a prospective series of such patients treated by subcapital cuneiform osteotomy and followed up for a mean of eight years and three months (2 years 5 months to 16 years 4 months). The incidence of complications is comparable with that reported for other surgical treatments for this subgroup. ${ }^{2}$ Patients with severe slips have problems of sitting and stooping, and an abnormal gait. In our study patients enjoyed an excellent range of hip movement and function. Those with grade 1 degenerative changes enjoyed good function with high scores, but it is important to identify these patients and to follow them since they are at risk of further progression. Two hips ( $8 \%$ ) showed significant grade 2 radiological changes and reduction in joint space at follow-up. This was associated with AVN in one hip and chondrolysis in the other.

In regard to the long-term results and the development of osteoarthritis, Hansson et $\mathrm{al}^{20}$ reported on the treatment of 59 hips by nailing in situ and showed that at a mean followup of 30.9 years, nine $(22 \%)$ of 41 patients with slips $\leq 30^{\circ}$ had developed degenerative changes with narrowing of the joint space ( 8 mild and 1 severe) as did nine $(50 \%)$ of 18 patients with slips between $31^{\circ}$ and $50^{\circ}(6$ mild and 3 severe). There were no slips greater than $50^{\circ}$ and the authors stated that long-term studies were required to decide on which method of treatment would be appropriate for such slips. From their data it can be inferred that fixation in situ for severe slips would yield a higher percentage with degenerative change. Our study shows that 23 (92\%) of 25 hips with a severe slip had a normal joint space at a mean of follow-up of 8 years 3 months. However, this is too short a period to allow comment on whether further degenerative change will develop. Fish ${ }^{12}$ studied 66 slips treated by cuneiform osteotomy at a mean of 13 years and four months and found osteoarthritis to have developed in six $(9 \%)$, which is comparable to the findings of our group.

Our results compare favourably with those of the longterm follow-up reported by Carney et al. ${ }^{2}$ They stated that the natural history of a malunited slip is one of deterioration related to the severity of the slip and complications. At a mean follow-up of 41 years, the Iowa hip score and radiological scores worsened with increasing severity of slip even when reduction or realignment had been performed. At the latest follow-up their severe chronic slips had a rate of AVN of $19 \%$ and of chondrolysis of $31 \%$ with a radiological degenerative grade of 2.4. When considering the method of treatment in chronic slips, those which had an osteotomy had a rate of AVN of $14 \%$, and of chondrolysis of $38 \%$, with a radiological degenerative grade of $2.5 .^{2}$ Our results had a lower rate of complications and a mean radiological degenerative grade of 0.3 , and we believe that by anatomically correcting the deformity in severe slips, we have potentially altered the natural history of the process for the better. However, this has yet to be determined by a longer period of follow-up.

No benefits in any form have been received or will be received from a commercial party related directly or indirectly to the subject of this article.

\section{References}

1. Mooney JF 3rd, Sanders JO, Browne RH, et al. Management of unstable/acute slipped capital femoral epiphysis: results of a survey of the POSNA membership. $\checkmark$ Pediatr Orthop 2005;25:162-6.

2. Carney BT, Weinstein SL, Noble J. Long-term follow-up of slipped capital femoral epiphysis. J Bone Joint Surg [Am] 1991;73-A:667-74.

3. Boyer DW, Mickelson MR, Ponseti IV. Slipped capital femoral epiphysis: Iongterm follow-up study of one hundred and twenty-one patients. J Bone Joint Surg [Am] 1981;63-A:85-95.

4. Loder RT. Unstable slipped capital femoral epiphysis. J Pediatr Orthop 2001;21: 694-9.

5. Carney BT, Weinstein SL. Natural history of untreated chronic slipped capital femoral epiphysis. Clin Orthop 1996;322:43-7.

6. Ross PM, Lyne ED, Morawa LG. Slipped capital femoral epiphysis: long-term results after 10-38 years. Clin Orthop 1979;141:176-80. 
7. Oram V. Epiphysiolysis of the head of the femur: a follow-up examination with special reference to end results and the social prognosis. Acta Orthop Scand 1953;23: 100-20.

8. Rattey T, PiehI F, Wright JG. Acute slipped capital femoral epiphysis: review of outcomes and rates of avascular necrosis. J Bone Joint Surg [Am]1996;78-A:398-402.

9. Murray R0. The aetiology of primary osteoarthritis of the hip. Radio/1965;38:810-24.

10. Stulberg SD, Cordell LD, Harris WH, Ramsey PL, MacEwen G. Unrecognized childhood hip disease: a major cause of idiopathic osteoarthritis of the hip. In: Procs third open scientific meeting of the Hip Society. C. V. Mosby, 1975:212-28.

11. Dunn DM, Angel JC. Replacement of the femoral head by open operation in severe adolescent slipping of the upper femoral epiphysis. J Bone Joint Surg [Br]1978;60-B: 394-403.

12. Fish JB. Cuneiform osteotomy of the femoral neck in the treatment of slipped capital femoral epiphysis: a follow-up note. J Bone Joint Surg [Am] 1994;76-A:46-59.

13. Abraham E, Garst J, Barmada R. Treatment of moderate to severe slipped capital femoral epiphysis with extracapsular base-of-neck osteotomy. J Pediatr Orthop 1993; 13:294-302.

14. Kramer WG, Craig WA, Noel S. Compensating osteotomy at the base of the femoral neck for slipped capital femoral epiphysis. J Bone Joint Surg [Am] 1976;58-A: 796-800.

15. Southwick W0. Osteotomy through the lesser trochanter for slipped capital femoral epiphysis. J Bone Joint Surg [Am] 1967;49-A:807-35.

16. Kartenbender K, Cordier W, Katthagen BD. Long-term follow-up study after corrective Imhauser osteotomy for severe slipped capital femoral epiphysis. J Pediatr Orthop 2000;20:749-56.

17. Loder RT, Richards BS, Shapiro PS, Reznick LR, Aronsson DD. Acute slipped capital femoral epiphysis: the importance of physeal stability. J Bone Joint Surg [Am] 1993;75-A:1134-40

18. Larson CB. Rating scale for hip disabilities. Clin Orthop 1963;31:85-93.

19. Harris WH. Traumatic arthritis of the hip after dislocation and acetabular fractures: treatment by mold arthroplasty: an end-result study using a new method of result evaluation. J Bone Joint Surg [Am] 1969;51-A:737-55

20. Hansson G, Billing L, Hogstedt B, Jerre R, Wallin J. Long-term results after nailing in situ of slipped upper femoral epiphysis: a 30-year follow-up of 59 hips. J Bone Joint Surg [Br] 1998;80-B:70-7.

21. Watson-Jones R. Fractures of the neck of femur. Brit J Surg 1936;23:787-808.

22. Griffith MJ. Slipping of the capital femoral epiphysis. Ann R Coll Surg Eng/1976;58: 34-42.

23. Rang M. Anthology of orthopaedics. Edinburgh: E \& S Livingstone 1996:139-43.
24. Kennedy JG, Hresko MT, Kasser JR, et al. Osteonecrosis of the femoral head associated with slipped capital femoral epiphysis. J Pediatr Orthop 2001;21:189-93.

25. Peterson MD, Weiner DS, Green NE, Terry CL. Acute slipped capital femoral epiphysis: the value and safety of urgent manipulative reduction. J Pediatr Orthop 1997; 17:648-54.

26. Aronsson DD, Loder RT. Treatment of the unstable (acute) slipped capital femoral epiphysis. Clin Orthop 1996;322:99-110.

27. Hall JE. The results of treatment of slipped femoral epiphysis. J Bone Joint Surg [Br] 1957;39-B:659-73.

28. Wilson PD, Jacobs B, Schecter L. Slipped capital femoral epiphysis: an end-result study. J Bone Joint Surg [Am] 1965;47-A:1128-45.

29. Hansson LI, Hägglund G, Ordeberg G. Slipped capital femoral epiphysis in southern Sweden 1910-1982. Acta Orthop Scand 1987;226(suppl):1-67.

30. Rooks MD, Schmitt W, Drvaric DM. Unrecognized pin penetration in slipped capital femoral epiphysis. Clin Orthop 1988;234:82-9.

31. Swiontkowski M. Slipped capital femoral epiphysis: complication related to internal fixation. Orthopedics 1983;6:705-12.

32. O'Brien ET, Pahey JJ. Remodeling of the femoral neck after in situ pinning for slipped capital femoral epiphysis. J Bone Joint Surg [Am] 1977;59-A:62-8.

33. Bellemans J, Fabry G, Molenaers G, Lammens J, Moens P. Slipped capital femoral epiphysis: a long-term follow-up. with special emphasis on the capacities for remodeling. J Pediatr Orthop B 1996;5:151-7.

34. Siegel DB, Kasser JR, Sponseller P, Gelberman RH. Slipped capital femoral epiphysis: a quantitative analysis of motion, gait, and femoral remodeling after in situ fixation. J Bone Joint Surg [Am] 1991;73-A:659-66.

35. Gage JR, Sundberg AB, Nolan DR, Sletten RG, Winter RB. Complications after cuneiform osteotomy for moderately or severely slipped capital femoral epiphysis. J Bone Joint Surg [Am] 1978;60-A:157-65.

36. Clarke HJ, Wilkinson JA. Surgical treatment for severe slipping of the upper femoral epiphysis. J Bone Joint Surg [Br] 1990;72-B:854-8.

37. DeRosa GP, Mullins RC, Kling TF Jr. Cuneiform osteotomy of the femoral neck in severe slipped capital femoral epiphysis. Clin Orthop 1996;322:48-60.

38. Phillips SA, Griffiths WEG, Clarke NM. The timing of reduction and stabilisation of the acute, unstable, slipped upper femoral epiphysis. J Bone Joint Surg [Br]2001; 83-B:1046-9.

39. Jackson-Burrows H. Slipped upper femoral epiphysis: characteristics of 100 cases. J Bone Joint Surg [Br] 1957;39-B:641-58.

40. Newman PH. The surgical treatment of slipping of the upper femoral epiphysis. J Bone Joint Surg [Br] 1960;42-B:280-8. 